\title{
AD HOC CONSULTANTS - 2020
}

Adolfo Jesiel Siebra - Universidade Federal do Ceara, Fortaleza-CE, Brasil

Alessandra Ackel Rodrigues - Centro Universitário Barão de Mauá, Ribeirão Preto-SP, Brasil

Alessandra Gotuzo Seabra - Universidade Presbiteriana Mackenzie, São Paulo-SP, Brasil

Alessandra Turini Bolsoni-Silva - Universidade Estadual Paulista, Bauru-SP, Brasil

Alexsandro Luiz de Andrade - Universidade Federal do Espirito Santo, Vitória-ES, Brasil

Amanda Londero-Santos - Universidade Federal do Rio de Janeiro, Rio de Janeiro-RJ, Brasil

Amanda Pinheiro Said - Universidade de Brasília, Brasília-DF, Brasil

Ana Cristina Resende - Pontifícia Universidade Católica de Goiás, Goiânia-GO, Brasil

Angela Helena Marin - Universidade Federal do Rio Grande do Sul, Porto Alegre-RS, Brasil

Antonio Abel Pires - Universidade do Porto, Porto, Portugal

Berta Rodrigues Maia - Universidade Católica Portuguesa, Braga, Portugal

Bruno Figueiredo Damásio - Universidade Federal do Rio de Janeiro, Rio de Janeiro-RJ, Brasil

Camila Rocha de Oliveira - Centro Brasileiro de Análise e Planejamento, São Paulo-SP, Brasil

Carina Nunes Bossardi - Universidade do Vale do Itajaí, Itajaí-SC, Brasil

Carlo Schmidt - Universidade Federal de Santa Maria, Santa Maria-RS, Brasil

Carlos Eduardo Norte - Universidade do Estado do Rio de Janeiro, Rio de Janeiro-RJ, Brasil

Cibelle Kayenne Martins Roberto Formiga - Universidade Estadual de Goiás, Goiânia-GO, Brasil

Claudia Maria Simões Martinez - Universidade Federal de São Carlos, São Carlos-SP, Brasil

Clarissa Mendonça Corradi-Webster - Universidade de São Paulo, Ribeirão Preto-SP, Brasil

Clarissa Pinto Pizarro de Freitas - Universidade Salgado de Oliveira, Niterói-RJ, Brasil

Clarissa Tochetto de Oliveira - Universidade Federal do Rio Grande do Sul, Porto Alegre-RS, Brasil

Cristian Zanon - Universidade Federal do Rio Grande do Sul, Porto Alegre-RS, Brasil

Cynthia de Freitas Melo - Universidade de Fortaleza, Fortaleza-CE, Brasil

Daniel Bartholomeu - Centro Universitário FIEO, Osasco-SP, Brasil

Danilo Ramos - Universidade Federal do Paraná, Curitiba-PR, Brasil

Dantielli Assumpção Garcia - Universidade Estadual do Oeste do Paraná, Cascavel-PR, Brasil

Denise Falcke - Universidade do Vale do Rio dos Sinos, São Leopoldo-RS, Brasil

Diene Monique Carlos - Universidade Federal de São Carlos, São Carlos-SP, Brasil 
Eda Marconi Custódio - Universidade de São Paulo, São Paulo-SP, Brasil

Eduardo Name Risk - Universidade Federal de São Carlos, São Carlos-SP, Brasil

Erich Montanar Franco - Universidade Presbiteriana Mackenzie, São Paulo-SP, Brasil

Erika Tiemi Kato Okino - Universidade de São Paulo, Ribeirão Preto-SP, Brasil

Evani Zambon Marques da Silva - Pontifícia Universidade Católica de São Paulo, São Paulo-SP, Brasil

Evanisa Helena Maio de Brum - Centro Universitário CESMAC, Maceió-AL, Brasil

Fabiana Queiroga - Centro Universitário de Brasília, Brasília-DF, Brasil

Fernanda Aguiar Pizeta - Universidade Paulista, Ribeirão Preto-SP, Brasil

Fernanda Pessolo Rocha - Centro Universitário Barão de Mauá, Ribeirão Preto-SP, Brasil

Flávia Helena P. Padovani - Universidade Estadual Paulista, Botucatu-SP, Brasil

Francirosy Campos Barbosa - Universidade de São Paulo, Ribeirão Preto-SP, Brasil

Gimol Benzaquen Perosa - Universidade Estadual Paulista, Botucatu-SP, Brasil

Gisele Cristina Resende - Universidade Federal do Amazonas, Manaus-AM, Brasil

Graziela Sousa Nogueira - Secretaria de Estado da Saúde do Distrito Federal, Brasília-DF, Brasil

Gustavo Martileli Massola - Universidade de São Paulo, São Paulo-SP, Brasil

Heloísa Ferreira - Universidade Federal do Triangulo Mineiro, Uberaba-MG, Brasil

Igor Londero - Universidade Federal do Rio Grande do Sul, Porto Alegre-RS, Brasil

Jana Gonçalves Zappe - Universidade Federal de Santa Maria, Santa Maria-RS, Brasil

Janine Kieling Monteiro - Universidade do Vale do Rio dos Sinos, São Leopoldo-RS, Brasil

Jorge Luiz da Silva - Universidade de Franca, Franca-SP, Brasil

José Aloyseo Bzuneck - Universidade Estadual de Londrina, Londrina-PR, Brasil

José Egídio Oliveira - Escola Superior de Saúde do Alcoitão, Alcabideche, Portugal

Laísy de Lima Nunes - Universidade Federal de Rondônia, Porto Velho-RO, Brasil

Laura Cristina Eiras Coelho Soares - Universidade Federal de Minas Gerais, Belo Horizonte-MG, Brasil

Leandro Castro Oltramari - Universidade Federal de Santa Catarina, Florianópolis-SC, Brasil

Leandro Silva Almeida - Universidade do Minho, Braga, Portugal

Luciane da Rosa Piccolo - Universidade Federal do Rio Grande do Sul, Porto Alegre-RS, Brasil

Lucila Moraes Cardoso - Universidade Estadual do Ceara, Fortaleza-CE, Brasil

Maíra Stivaleti Colombarolli - Universidade de São Paulo, Ribeirão Preto-SP, Brasil 
Manoel Antônio dos Santos - Universidade de São Paulo, Ribeirão Preto-SP, Brasil

Marcelo Gustavo Aguilar Calegare - Universidade Federal do Amazonas, Manaus-AM, Brasil

Márcia Maria Peruzzi Elia da Mota - Universidade do Estado do Rio de Janeiro, Rio de Janeiro-RJ, Brasil

Maria Manuela Martins - Escola Superior de Enfermagem do Porto, Porto, Portugal

Maria Nivalda Carvalho-Freitas - Universidade Federal de São João del-Rei, São João del Rei-MG, Brasil

Mariane Ricardo Acosta Lopez Molina - Universidade Católica de Pelotas, Pelotas-RS, Brasil

Marina Kohlsdorf - Centro Universitário UNICEUB, Brasília-DF, Brasil

Marina Zanella Delatorre - Universidade Federal do Rio Grande do Sul, Porto Alegre-RS, Brasil

Miriam Raquel Wachholz Strelhow - Universidade de São Paulo, São Paulo-SP, Brasil

Miriam Tachibana - Universidade Federal de Uberlândia, Uberlândia-MG, Brasil

Myriam Aparecida Mandetta Pettengill, Universidade Federal de São Paulo, São Paulo-SP, Brasil

Nadia Maria Ribeiro Salomão - Universidade Federal da Paraíba, João Pessoa-PB, Brasil

Neire Aparecida Machado Scarpini - Centro Universitário Moura Lacerda, Ribeirão Preto-SP, Brasil

Nelson Hauck Filho - Universidade São Francisco, Campinas-SP, Brasil

Odair Sass - Pontifícia Universidade Católica de São Paulo, São Paulo-SP, Brasil

Paloma Pegolo de Albuquerque - Universidade Federal do Triângulo Mineiro, Uberaba-MG, Brasil

Patricia Alvarenga - Universidade Federal da Bahia, Salvador-BA, Brasil

Patricia Lorena Quiterio - Universidade do Estado do Rio de Janeiro, Rio de Janeiro-RJ, Brasil

Patricia Martins Freitas - Universidade Federal da Bahia, Vitória da Conquista-BA, Brasil

Paula Inez Cunha Gomide - Universidade Tuiuti do Paraná, Curitiba-PR, Brasil

Paula Orchiucci Miura - Universidade Federal de Alagoas, Maceió-AL, Brasil

Pedro Paulo Pires dos Santos - Universidade Federal do Rio de Janeiro, Rio de Janeiro-RJ, Brasil

Rafaela Silva Moreira - Universidade Federal de Santa Catarina, Florianópolis-SC, Brasil

Rejane Rosaria Grecco dos Santos - Universidade Federal do Rio Grande, Pelotas-RS, Brasil

Rodrigo Rezende Ferreira - Universidade de Brasília, Brasília-DF, Brasil

Sabrina Martins Barroso - Universidade Federal do Triângulo Mineiro, Uberaba-MG, Brasil

Silvia Renata Magalhães Lordello - Universidade de Brasília, Brasília-DF, Brasil

Sofia Santos - Universidade de Lisboa, Lisboa, Portugal

Sônia Maria Guedes Gondim - Universidade Federal da Bahia, Salvador-BA, Brasil 
Paidéia, 30, e3041

Sonia Regina Pasian - Universidade de São Paulo, Ribeirão Preto-SP, Brasil

Susana Maria Gonçalves Coimbra - Universidade do Porto, Porto, Portugal

Susana Nunez Rodriguez - Universidade Salgado de Oliveira, Niterói-RJ, Brasil

Suzane Carvalho da Vitória Barros - Instituto Nacional de Câncer, Niterói-RJ, Brasil

Vanessa Rissi - IMED, Passo Fundo-RS, Brasil

Vicente Cassepp-Borges - Universidade Federal Fluminense, Niterói-RJ, Brasil

William Sorensen - University of Texas at Tyler, Texas, USA 